\title{
Experimental assessment of exposure to gaseous pollutants from mattresses and pillows while asleep.
}

\author{
Jelle Laverge ${ }^{1, *}$, Atila Novoselac ${ }^{2}$, Richard Corsi ${ }^{2}$, Arnold Janssens ${ }^{1}$ (12 pt, style: Author) \\ ${ }^{1}$ Ghent University (UGent), Gent, Belgium \\ ${ }^{2}$ University of Texas at Austin (UT), Austin, Texas, USA \\ *Corresponding email: jelle.laverge@ugent.be
}

Keywords: inhalation, measurements, residential, sources/emissions, thermal plume

\section{Introduction}

On average, we spend about $1 / 3$ of our life asleep. This amounts to about $70 \%$ of the time we spend at home (Basner, 2007; Glorieux, 2008). The exposure conditions in the bedroom are fundamentally different than in other rooms, mainly due to the fact that for a large majority of time spent in the bedroom, the occupants are asleep, with their head pressed against some of the major potential emission sources in the bedroom: mattress, bed cloth, and pillow. Mattresses, pillows and bed linens are often heavily treated with flame-retardants, and may also be sources of phthalates, isocyanates, formaldehyde, detergent components, and other substances that are known to have an impact on human health. While asleep, the nose and mouth are in direct proximity of these sources for a long period of time. Therefore, traditional exposure assessments based on the well-mixed room assumption are not applicable. In this paper, results of climate chamber experiments involving the use of a breathing thermal manikin are presented in which the impact of this proximity on the exposure is assessed.

\section{Methods}

In most ventilation and exposure studies, the room is assumed to be well mixed and the perfect mixing concentration is used to predict exposure. Likewise, carbon dioxide controlled ventilation systems are based on the concentration measured by a sensor that is, at best, positioned to represent the well-mixed pollutant concentration. However, the real concentration of the inhaled air will, due to the proximity of source and sleeping person, be higher in the case of emissions from a mattress.

To quantify such exposures, experimental measurements in a test room with a breathing thermal manikin were conducted. The test room is an airtight stainless steel climate chamber with internal dimensions of $5.5 \times 4.5 \times 2.7 \mathrm{~m}^{3}$. The mechanical system of the climate chamber controlled the indoor environment. Temperature was in the range of $24 \pm 1^{\circ} \mathrm{C}$ and the only source of heat in the room was the thermal manikin positioned in the sleeping position on a bed.

The chamber was ventilated at a rate of 0.5 air changes per hour (ACH). There was no air recirculation and the supply air was all fresh air. The air was supplied to the room with a low velocity diffuser sitting on the ground. The supply velocity was below $0.05 \mathrm{~m} / \mathrm{s}$, which prevented formation of supply jet and air mixing due to the ventilation flow. The exhaust vent was on a side wall of the chamber, just beneath the ceiling.

The breathing thermal manikin (Donghyun, 2010; Rim, 2009; Rim, 2008) was positioned on a twin size mattress on a bedframe, with the headboard against the centre of the longer wall of the chamber on the opposite side from the exhaust. The manikin's breathing system was adjusted for inhalation through the nose during sleep. Minute ventilation was $6 \mathrm{~L} / \mathrm{min}$ and the respiratory rate was 12 breaths $/ \mathrm{min}$, consistent with observations made in sleeping subjects (Krieger, 2005). The metabolic heat load of the manikin was set to $70 \mathrm{~W}$ of sensible heat.

A tracer $\left(0.1 \% \mathrm{SF}_{6}\right.$ in $\mathrm{N}_{2}$ gas) was used as a surrogate for any pollutants emitted from the mattress in the gas phase. A multi-point distribution system was built into the mattress so that these items in the immediate vicinity of the manikin acted as uniform emission sources. The $\mathrm{SF}_{6}$ concentration in the inhaled air was measured using GC-ECD. 
The manikin was positioned in a way that corresponds to 3 different common sleeping positions: (1) supine, i.e., manikin positioned on its back on the centerline of the bed, with arms positioned on the side of the torso, (2) lateral, i.e., manikin lying on its right side, with the arms held along side the torso and a $90^{\circ}$ forward angle at the elbow, and (3) prone, i.e., manikin turned on the chest with one arm positioned along the torso and the other positioned upward with the left hand next to the head.

\section{Results and Discussion}

The results are expressed as intake fractions. Since $\mathrm{SF}_{6}$ is used as a tracer and could represent any gas phase emission from the source, only the relative difference between the measured intake fraction and the intake fraction calculated based on the well-mixed concentration is relevant. Intake fractions measured for the supine position were $24 \%$ higher than those calculated based on an assumed well-mixed test room. For the lateral and prone positions, the measured increase in intake fraction relative to a well-mixed room were 40 and $70 \%$, respectively.

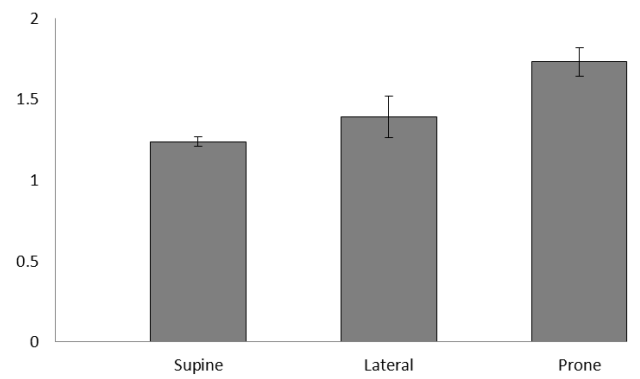

Figure 1. relative intake fractions for 3 positions

The thermal plume produced by the heated manikin proved to play an essential role in the determination of the air flow pattern and is the dominant driving force in the dilution of the $\mathrm{SF}_{6}$ tracer. This is clearly demonstrated by an additional case when the heat source in the manikin was shut off, and the intake fraction increased by $80 \%$ compared to the case with the heat source activated. This amounts to a $120 \%$ increase compared to the intake fraction calculated based on a well-mixed concentration. Increasing the mixing in the room by adding fans did not change the relative intake fraction compared to the well-mixed case.
Some people tend to sleep with their head completely covered by bed linens. As the thermal plume is shown to be the dominant factor in the dilution of emissions in the proximity of the sleeping person, this habit can be expected to have a large impact on the intake fraction. An additional case in the supine position under these circumstances showed a more than 20 fold $(2400 \%)$ increase in intake fraction compared to the normal supine case.

\section{Conclusions}

This paper presents the results from climate chamber tests with a breathing thermal manikin that assess the intake fraction for nose breathing for gaseous emissions from mattresses and pillows. The intake fractions for several sleep positions as well as different bedding arrangements are reported. The results demonstrate that human metabolism is a dominant factor in the dilution of emissions in close proximity of the nose, reducing exposure by $40 \%$ compared to a case without metabolic heat output. This effect is more important than the sleep position. Sleeping with the head under the covers increases intake by a factor 24 .

\section{References}

Basner, M., Fomberstein, K.M., Razavi, F.M., Banks, S., William, J.H., Rosa, R.R. and Dinges, D.F. (2007) American time use survey: Sleep time and its relationship to waking activities, Sleep, volume 30, 10851095.

Donghyun, R. and Novoselac, A. (2010) Occupational Exposure to Hazardous Airborne Pollutants: Effects of Air Mixing and Source Location, Journal of Occupational and Environmental Hygiene, volume 7, 683-692692.

Glorieux, I.M., J. (2008) Belgisch tijdsbudgetonderzoek, Vol. 2010, Brussels.

Krieger, J. (2005) Respiratory physiology: breathing in normal subjects, In: Kryger, M. H. (ed) Principles and practise of sleep medicine, Elsevier Saunders.

Rim, D. and Novoselac, A. (2008) Inhalation Exposure and Accessibility of Source in the Vicinity of Human Body, Epidemiology, volume 19, S353-S353.

Rim, D. and Novoselac, A. (2009) Transport of particulate and gaseous pollutants in the vicinity of a human body, Building and Environment, volume 44, 1840-1849. 